\title{
NARRATIVAS DEL COLECTIVO LA COMADRE SOBRE LA ESTRATEGIA DE GENERACIÓN DE INGRESOS EN EL DISTRITO DE CARTAGENA
}

\section{NARRATIVES OF THE COLLECTIVE "LA COMADRE" ON THE STRATEGY OF INCOME GENERATION IN THE DISTRICT OF CARTAGENA}

Ana Milena Álvarez-Hernández ${ }^{1}$; Ginna Paola Pérez-Castro ${ }^{2}$; Damarys Villarreal-Gómez ${ }^{3}$; Alejandro Villarreal-Gómez ${ }^{4}$ (D) .

1. Trabajadora Social. Universidad de Cartagena. anamilena-alvarez@hotmail.com. 2. Antropóloga. Universidad de Cartagena. Docente. ginna2210@gmail.com

3. Profesional en Desarrollo Comunitario. Trabajo Social.Universidad de Cartagena. damarisvillareal@hotmail.com 4. Administrador de Empresa. Universidad de Cartagena. avillarrealg@unicartagena.edu.co

*Correspondencia del Autor: Damarys Villarreal-Gómez, correo electrónico: damarisvillareal@hotmail.com.

\section{RESUMEN}

Este artículo presenta algunos resultados y reflexiones de la investigación "Percepciones del Colectivo "La Comadre" en torno a la estrategia de generación de ingresos de la política pública de víctimas del desplazamiento en la ciudad de Cartagena de Indias", cuyo objetivo principal fue analizar las percepciones construidas por las mujeres Afrocolombianas del colectivo "La Comadre" en torno a la estrategia de generación de ingresos de la Política Pública de Víctimas de Desplazamiento Forzado implementada en el Distrito de Cartagena de Indias, para la identificación de los desafíos actuales sobre la articulación entre las políticas públicas, organizaciones sociales y la gobernanza en el territorio.

De esta forma, el proceso de investigación se trabajó desde un enfoque cualitativo y un paradigma hermenéutico y fenomenológico que permitió realizar un acercamiento y análisis a las percepciones construidas por el colectivo y a su vez, se identificó desde las narrativas de las mujeres y las voces de los funcionarios públicos y académicos las apuestas para el fortalecimiento de políticas públicas en relación a la generación de ingresos para las mujeres afrocolombianas.

En este sentido, conocer las perspectivas de las mujeres, de los funcionarios públicos y los académicos permitió evidenciar la inconformidad con relación a las acciones encaminadas a la generación de ingresos para las mujeres afrocolombianas desplazadas; así mismo, desde sus narrativas, se afirma que existen grandes desafíos para el Distrito de Cartagena relacionados con avances significativos en términos de implementación y cumplimiento de políticas públicas orientadas a la generación de ingresos.

Palabras claves: Percepciones; desplazamiento forzado; víctimas del conflicto armado; políticas públicas; generación de ingresos.

Cómo citar:

Álvarez-Hernández, Ana Milena; Pérez-Castro, Ginna Paola; Villarreal-Gómez, Damarys; Villarreal-Gómez, Alejandro. (2021). Narrativas del colectivo la comadre sobre la estrategia de generación de ingresos en el distrito de Cartagena. Revista de Investigaciones Universidad del Quindio, 33(1), 44-57. https://doi.org/10.33975/riuq.vol33n1.443 


\begin{abstract}
The article presents some results and reflections of the research "Perceptions of the Collective "La Comadre" around the strategy of generating income of the public policy of displacement victims in the city of Cartagena", whose main objective was to analyze the perceptions built by Afro-Colombian women of the collective La Comadre around the revenue generation strategy of the Public Policy of Victims of Forced Displacement implemented in the District of Cartagena de Indias, to identify current challenges on the articulation between public policies, social organizations and governance in the territory.

In this way, the research process was worked from a qualitative approach and a hermeneutic and phenomenological paradigm that allowed to carry out an approach and analysis to the perceptions built by the Collective and, in turn, was identified from the narratives of women and the voices of public and academic officials bets on the strengthening of public policies in relation to the generation of income for AfroColombian women.

In this regard, knowing the perspectives of women, civil servants and academics showed nonconformity in relation to income-generating actions for displaced AfroColombian women; likewise, from its narratives, it is stated that there are major challenges for the District of Cartagena related to significant advances in terms of implementation and compliance with public policies oriented to revenue generation.
\end{abstract}

Keywords: Perceptions; forced displacement; victims of armed conflict; public policies; income generation.

\section{INTRODUCCIÓN}

El conflicto armado colombiano se configura como un hecho histórico que se ha caracterizado por ser uno de los más largos del mundo y por las graves violaciones a los derechos humanos, generando múltiples afectaciones individuales, familiares y sociales. (Rojas, 2016), conflicto que ha suscitado incontables manifestaciones y consecuencias, entre ellas el desplazamiento forzado, que de acuerdo con los postulados del (Centro Nacional de Memoria Histórica , 2015) "más de seis millones de personas se han visto forzadas a desplazarse dentro y fuera del territorio nacional, abandonando sus hogares, sus tierras y territorios, sus bienes, sus costumbres, sus comunidades y su forma de vida" (p. 25), desplazamiento que ha generado múltiples afectaciones económicas, sociales, políticas y culturales sobre la población colombiana.

En este sentido, y dentro de las múltiples representaciones que se confieren al desplazamiento forzado, se establece que ha marcado de manera significativa $y$ desproporcionadamente la vida de las niñas y las mujeres, quienes se han configurado históricamente como uno de los grupos sociales más vulnerables, en específico de las mujeres afrocolombianas, raizales y palenqueras que, de acuerdo con datos del registro único de víctimas (RUV, 2020) representan a nivel nacional el 28\% de la población en situación de desplazamiento, y el $9.3 \%$ de las mujeres víctimas del conflicto armado.

Ahora bien, la problemática del desplazamiento forzado no es ajena al Distrito de Cartagena, teniendo en cuenta que, la ciudad se visibiliza como una de las grandes ciudades destinataria de personas desplazas, de acuerdo con los planteamientos de (Puello, Romero, \& García, 2010) Cartagena se ha convertido en un territorio receptor de la población víctima de 
desplazamiento forzado por conflicto armado, en especial de los municipios que conforman el departamento de Bolívar en donde las dinámicas del conflicto y el desplazamiento provienen mayormente de la región de los Montes de María; asimismo, y de acuerdo con el (RUV, 2020) en la ciudad de Cartagena de Indias residen alrededor de 40.385 mujeres afrocolombianas víctimas del conflicto armado.

De esta forma, las mujeres afrodescendientes representan un grupo prioritario dentro de la población que deber ser atendida por el Estado, en este sentido, es fundamental tener en cuenta que los derechos de la población víctima de desplazamiento forzado y en especial los derechos de las mujeres afrodescendientes, se encuentran protegidos por una legislación que se enmarca a nivel internacional y nacional, promoviendo acciones afirmativas tendientes a garantizar la reparación y reivindicación de derechos a nivel individual y colectivo.

Por tal motivo, en el marco del desplazamiento forzado se han instaurado diferentes acciones tendientes a garantizar la protección y atención de la población desplazada, entre estas acciones se destaca la estrategia de generación de ingresos derivada de la Política Pública de Víctimas del Desplazamiento Forzado, la cual se debe encontrar articulada con los programas y planes de desarrollo en el contexto local, propendiendo por el desarrollo de líneas de acción claves para promover estrategias de generación de ingresos focalizadas específicamente para las mujeres afrodescendientes víctimas de desplazamiento forzado en el Distrito de Cartagena.

Asimismo, dentro de los aspectos fundamentales a destacar, es de carácter primordial señalar que acuerdo con el (PNUD , 2010) “en los últimos años el país ha visto cómo crecen y se conforman nuevas organizaciones de mujeres que generan un tejido social con miras a construir la paz" ( $p$. 6 ); entre estas nuevas organizaciones y colectivos que están surgiendo desde procesos organizativos autónomos a nivel de comunidad, se visibiliza significativamente la labor de La Coordinación de Mujeres Afrocolombianas Desplazadas en Resistencia -Colectivo La Comadre-, integrado por mujeres afrodescendientes pertenecientes a la Asociación Nacional de Afrodescendientes Desplazados (AFRODES) conformada por 127 organizaciones, entre ellas La Comadre, quienes desde su estructura organizativa se encuentran proyectadas por organizaciones constituidas a nivel nacional, regional, departamental y local.

En este sentido, los procesos organizativos desarrollados por el colectivo La Comadre les han permitido a las mujeres fortalecer su accionar como sujetas políticas a fin de proteger y restaurar los derechos de las mujeres afrocolombianas víctimas de desplazamiento forzado $\mathrm{y}$ de conflicto armado, consolidándose como sujetas de reparación colectiva.

"Nos hemos organizado como una coordinación horizontal para realizar acciones de incidencia que conduzcan a que el gobierno colombiano diseñe e implemente las políticas públicas y programas que garanticen con un enfoque étnico de género la reparación de los derechos que nos han sido vulnerados como consecuencia del conflicto armado interno" Lideresa del Colectivo La Comadre (Becerra, 2019)

De esta forma, las mujeres dentro de su trabajo colectivo han insistido persistentemente para que los programas o iniciativas de generación de ingresos dirigidas a víctimas de desplazamiento forzado respondan a sus características sociales, regionales y productivas; en este sentido, se han direccionado políticas públicas tendientes a transformar la situación y realidad de las mujeres afrodescendientes, comprendiéndose las políticas públicas desde los planteamientos de (Jiménez \& Ramírez, 2008) la política pública se puede entender como la orientación general que define el marco de actuación de los poderes públicos en la sociedad, sobre un determinado asunto. De manera más concreta, la política pública se presenta como un proceso de decisiones y acciones gubernamentales actuando directamente 
o a través de sus agentes, tendientes a resolver un asunto de interés público.

En esta línea, la política pública y su estrategia de generación de ingresos representan el conjunto de acciones dirigidas a la atención e intervención de la población victimas de desplazamiento por el conflicto armado y le apunta a generar una estabilidad y bienestar económico.

Se trata de una nueva política, con la cual se planea atender la problemática del desplazamiento para dar una respuesta efectiva a la crisis humanitaria que vive la población desplazada, corregir las falencias e incongruencias que han sido detectadas tanto por el propio Gobierno, la sociedad civil y la Corte Constitucional, asegurar la efectividad de los derechos de la población desplazada e igualmente, adoptar las medidas que sean necesarias para avanzar en la superación del estado de cosas inconstitucional. (SNAIPD, 2011, p. 34).

En este sentido, es importante señalar que las políticas públicas dirigidas hacia la generación de ingresos buscan estructurar responsabilidades en lo referente a la coordinación, planeación, ejecución presupuestal y atención a la población en materia de generación de ingresos, propiciando de esta forma desde su deber ser condiciones reales de igualdad de oportunidades; motivo por el cual, es fundamental precisar que las acciones instauradas en el orden nacional y departamental se deberían encontrar contextualizadas a nivel local mediante el Plan Integral Único (PIU) para la prevención y atención a la problemática del desplazamiento en el Distrito de Cartagena, Plan de Acción Territorial y el Plan de Desarrollo Distrital.

Enconcordanciaconlosplanteamientosanteriores, la investigación indagó por las percepciones que han construido las mujeres del Colectivo La Comadre, un grupo de mujeres afrocolombianas víctimas de desplazamiento forzado, por hechos ocurridos en el marco del conflicto armado en relación a las acciones establecidas en torno a la estrategia de generación de ingresos de la Política Pública de Víctimas del Desplazamiento, percepciones configuradas a partir de los procesos vividos desde sus experiencias de acuerdo a las estrategias impartidas en el Distrito de Cartagena; en esta línea, las narrativas de las mujeres, los funcionarios públicos y académicos posibilitaron realizar reflexiones y construir aprendizajes significativos desde las experiencias vividas por las mujeres afrocolombianas y la realidad social del Distrito de Cartagena en lo referente a la generación de ingresos.

\section{MATERIALES Y MÉTODOS}

\section{Diseño}

El diseño metodológico del proceso de investigación se desarrolló bajo un enfoque cualitativo que "asume la realidad subjetiva, dinámica $\mathrm{y}$ compuesta por multiplicidad de contextos. El enfoque cualitativo de investigación privilegia el análisis profundo y reflexivo de los significados subjetivos e intersubjetivos que forman parte de las realidades estudiadas" (Mata, 2019), de esta manera, el enfoque cualitativo permitió rescatar las voces y resaltar las narrativas de los actores sociales participantes, desde sus experiencias en relación a implementación de la estrategia de generación de ingresos en la ciudad de Cartagena; de esta manera, se fundamentó bajo los paradigmas hermenéutico y fenomenológico, debido a que su importancia metodológica se fundamenta en relación a que "son enfoques adecuados dentro de la investigación cualitativa, están centrados en las experiencias vividas, comportamientos, sentimientos, funcionamiento organizacional entre otros". (Morse 2003 como se citó en (Natalia \& Inciarte, 2012, p. 201)

\section{Participantes}

La muestra estuvo constituida por un total 67 actores participantes, distribuidos de la siguiente manera: 40 mujeres afrocolombianas integrantes del colectivo La Comadre en la ciudad de 
Cartagena, específicamente en el barrio Olaya Herrera sector 11 de noviembre y 27 funcionarios públicos y académicos que dentro de su ejercicio profesional se enmarcaran dentro de la temática abordada; en este sentido, para el desarrollo de la investigación la muestra se seleccionó a partir de un muestreo no probabilístico por conveniencia, de esta forma, este tipo de muestra también denomina intencional más allá de lograr una gran representatividad numérica para el desarrollo de la investigación se centró la representatividad teórica y contribuciones que los actores participantes aportaran en la investigación.

\section{Técnicas}

El proceso de recolección de información se realizó mediante las técnicas de: 31 entrevistas realizadas a 27 funcionarios públicos y académicos y 4 mujeres afrocolombianas del colectivo La Comadre; 40 encuestas efectuadas a mujeres del colectivo La Comadre y 3 grupos focales llevados a cabo mediante tres sesiones, en los cuales participaron 15 mujeres del colectivo.

De igual forma, el desarrollo de la investigación se apoyó en las técnicas de análisis documental que permitió fundamentar aspectos teóricos $\mathrm{y}$ conceptuales esenciales para la investigación y la observación participante con el propósito de constituir la interacción social entre las investigadoras y los actores sociales participantes en su contexto social inmediato.

\section{Análisis de las categorías}

En relación a los objetivos establecidos en la investigación se derivó y desarrolló el sistema categorial o las categorías de análisis que tuvieron el propósito de agrupar por campos u ordenadores congregando datos similares, tal como establece (Galeano 2014 como se citó en (Castillo, Segrera, \& León, 2018)

Es clasificar la información por categorías de acuerdo a criterios temáticos referidos a la búsqueda de significados. Es conceptuar con un término o expresión que sea claro e inequívoco, el contenido de cada unidad temática con el fin de clarificar, contrastar, interpretar, analizar y teorizar (pág. 53).

En este sentido, el análisis de las categorías se basó en la codificación e interpretación de los datos y relatos obtenidos relacionándolos con las categorías establecidas, tomando como punto o eje central las categorías de desplazamiento forzado, percepciones y generación de ingresos quienes establecen el cuerpo y sentido de la investigación, a su vez, para realizar el análisis de dichos elementos se recurrió directamente con el análisis de los datos e interpretación de los mismos en relación a la revisión literaria.

Ilustración 1. Matriz de categorías

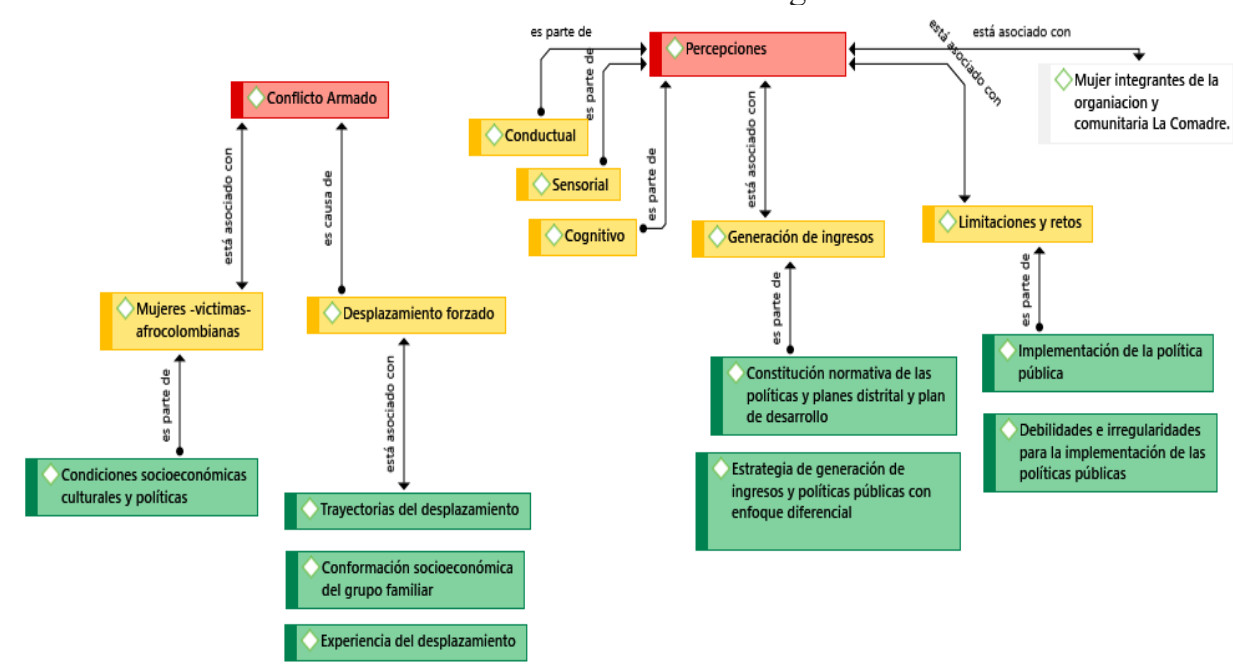

Fuente: elaborado por las autoras en Atlas Ti 


\section{Procesamiento de datos}

El procesamiento de la información cualitativa se realizó acudiendo al software Atlas Ti que permitió la codificación de la información obtenida mediante las entrevistas, asignando códigos y categorías a los relatos y narraciones para estructurar y organizar los hallazgos más representativos acorde con las categorías y descriptores de la investigación; a su vez, los datos cuantitativos obtenidos de las encuentras aplicas se trataron mediante la digitación y tabulación de los datos para su posterior representación gráfica y análisis.

\section{RESULTADOS}

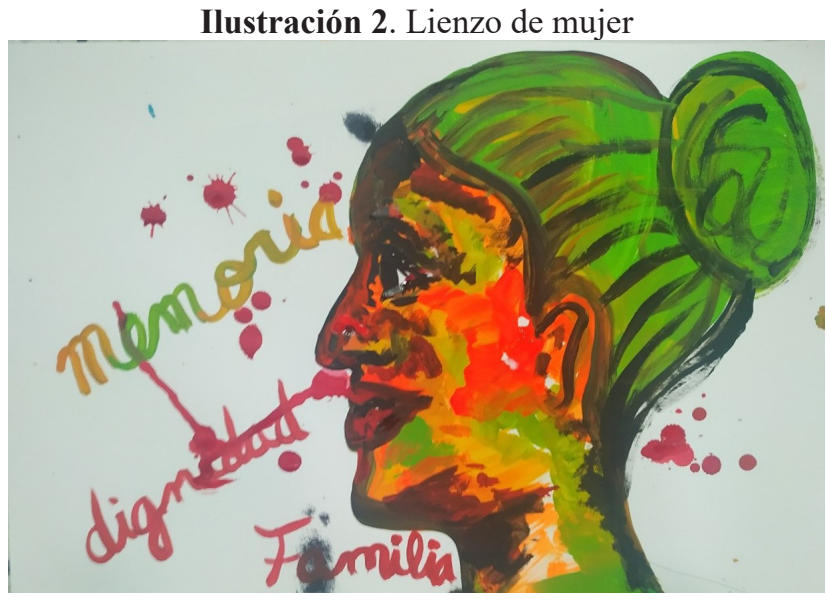

Fuente: Andrés Novoa

Incidencia política del Colectivo La Comadre ante la Corte Constitucional e implementación de acciones sobre estrategias de generación de ingresos en la ciudad de Cartagena

En primera instancia el proceso de investigación permitió un acercamiento a la realidad política que configura y da sentido a las acciones instauradas desde La Comadre, teniendo en cuenta que el colectivo proyecta su participación e incidencia política desde diversos espacios que se configuran hacia la promoción de los derechos constitucionales de las mujeres afrocolombianas, incidiendo de manera significativa en la normatividad gestada entre los años 2006 al 2020 desde el desarrollo de audiencias, encuentros regionales, autos y presentación de informes ante la Corte Constitucional.

Ilustración 3. Incidencia Política de La Comadre

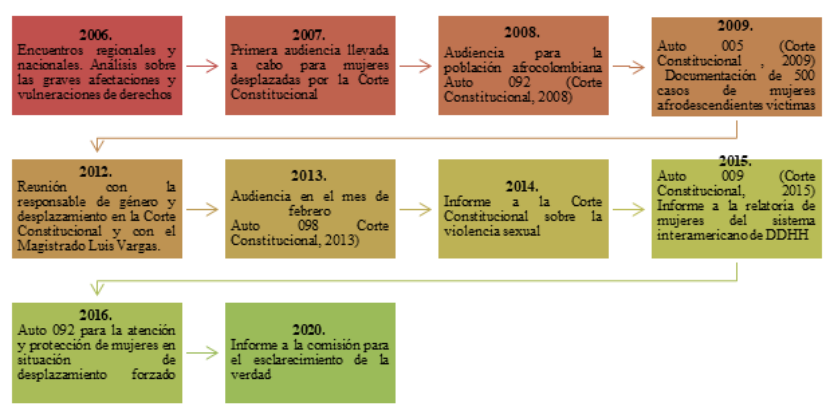

Fuente: elaborado por las autoras

De esta forma, las múltiples acciones emprendidas por el Colectivo La Comadre han permitido que a nivel nacional, departamental y distrital se evidenciaran las afectaciones particulares que vivieron las mujeres afrodescendientes en el marco del conflicto armado y el desplazamiento forzado, aspectos fundamentales que incidieron en el desarrollo y la visibilización para que la Corte Constitucional reconociera el impacto desproporcionado y de forma diferencial que presenta el conflicto armado y el desplazamiento forzado en las mujeres afrocolombianas víctimas. De igual manera, las acciones impartidas por el colectivo han permitido la incursión del enfoque de género a fin de abordar de manera diferencial la situación de las mujeres afrodescendientes.

"La naturaleza de La Comadre, y su especificidad como sujetas reparación colectiva, se puede entender a partir de los múltiples reconocimientos que sobre los daños experimentados ha ofrecido la Corte Constitucional, organismos internacionales como la Comisión Interamericana de Derechos Humanos, y numerosos reportes producidos por La Comadre y por AFRODES" Lideresa del Colectivo La Comadre (Becerra, 2019)

Ahora bien, referenciando y especificando la realidad y situación directa en la ciudad de Cartagena de Indias en cuanto a políticas y 
programas de gobierno direccionados a trabajar la intervención de población afrocolombiana en situación de desplazamiento forzado, desde el Distrito se han instaurado acciones que se consolidaron y aterrizaron en los siguientes apartados:

- El Plan Integral Único para la prevención y atención a la problemática del desplazamiento forzado en el Distrito de Cartagena (PIU) (Alcaldía de Cartagena, 2007)

- La adopción de la Política Pública con Enfoque Diferencial "Inclusión efectiva para la población afrocolombiana, negra, palenquera y raizal en el Distrito de Cartagena de Indias. Cartagena ciudad de oportunidades inclusivas".

- El Plan de Acción Territorial (PAT) 2016 - 2019 Víctimas Integradas. (Alcaldía de Cartagena , 2016)

En este sentido, es importante enfatizar que el PIU pretendía avanzar hacia el goce efectivo de los derechos de la población en situación de desplazamiento asentada en la ciudad de Cartagena de Indias a través de la consolidación de una política pública integral estructurada desde diferentes fases de intervención y líneas estratégicas para la prevención y la atención a la problemática, de esta forma, para el año 2005 en la ciudad se adelantaron acciones tendientes a garantizar el mandato ley conferido en la sentencia T-025 (Corte Constitucional, 2004); de esta manera, entre las tres fases que se desprendían de este plan se encuentran:

- Fase de prevención y atención,

- Fase de atención humanitaria en emergencia,

- Fase de estabilización socioeconómica, esta última enfatizó su accionar desde líneas estrategias orientadas hacia las áreas de salud, vivienda, educación y generación de ingresos, proyectándose desde procesos y acciones prioritarias.

De esta forma, el PIU se basó en el decreto 2050 (Congreso de la República , 2005) que definía unos propósitos para promover acciones y medidas a mediano y largo plazo, bajo los principios de concurrencia, complementariedad y subsidiariedad que para la época señalaba la ley; así mismo, se ejecutaron acciones inmediatas integrándose entidades que manejaban la problemática del desplazamiento, llevándose a cabo diferentes acciones, entre ellas una rueda de empresas privadas que se comprometieron a brindar oportunidades en torno a ser empleados, construcción de alberges para la atención de necesidades inmediatas como alimentación, aseo, manejo de abastecimiento, entre otras.

\section{Percepciones de La Comadre sobre la estrategia de ge- neración de ingresos de la política pública de victimas del desplazamiento implementada en el Distrito de Car- tagena}

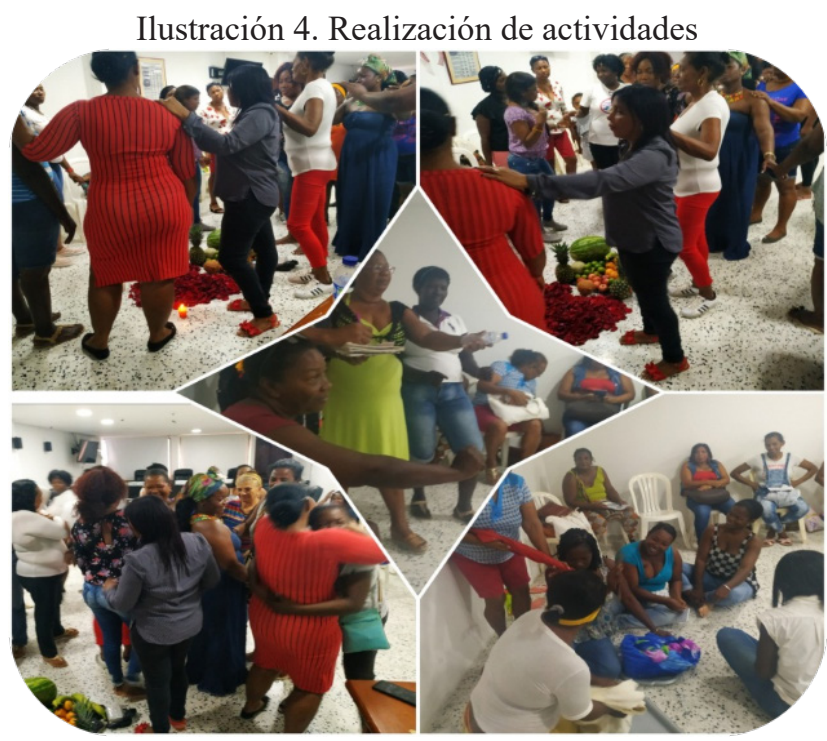

Fuente: autoras de la investigación

Ahora bien, al indagar sobre las percepciones que tienen las mujeres del colectivo La Comadre con relación a estrategia de generación de ingresos conferida desde la Política Pública de Víctimas de Desplazamiento Forzado, estas se configuran a partir de los significados y sentidos asociados desde sus experiencias; en este sentido, se evidencia la inconformidad de las mujeres afrodescendientes con relación a las acciones orientadas a la generación de ingresos para las víctimas de desplazamiento, destacando los siguientes aspectos: 
En primera instancia se visibiliza la poca efectividad de la política pública, asumiendo que, como mujeres afrodescendientes no se han sentido beneficiadas o incluidas en los procesos y escenarios de acción de la misma, por lo tanto, no evidencian avances en el Distrito con relación a la implementación de la política pública que promueva e incentive la generación de ingresos en las mujeres afrodescendientes asentadas en la ciudad de Cartagena.

"En términos de generación de ingresos no ha habido ningún avance, porque cuando realmente a las víctimas y a la población afrodescendiente se le ha llamado para darle un proyecto de fortalecimiento de negocios, son unos proyectos que realmente no cumplen con el objetivo que nosotros como víctimas queremos" E3LD_MlaComadre.

"Creo que hay muchas dudas sobre la temática porque la atención a la población víctima del desplazamiento ha sido muy paleativa con la entrega de mercados y así, pero un proceso de acompañamiento de permita dar el salto a la estabilización económica y a la generación de ingresos ha sido muy difícil" GF2_MLaComadre.

De esta manera, las mujeres evidencian y visibilizan una desarticulación de las apuestas estatales y las acciones llevadas a cabo en los contextos locales, debido a que las experiencias de las mujeres reflejan falencias en la participación, el informe presupuestal de la política de atención a la población víctima de desplazamiento, manifestó que durante el año 2019 uno de los aspectos priorizados fue: Participación de la mujer en las medidas asociadas a la Ley 1448 de 2011. (DNP, 2020).

Así mismo, evidenciaron una dicotomía presente en el enfoque transversal desde el que se plantea la política, desde este aspecto las mujeres destacan que, si bien la política se constituye desde un enfoque transversal y que los distintos aspectos de la población víctima de conflicto como un accionar general, las particularidades que presentan las mujeres afrodescendientes no pueden ser abordadas de manera generalizada.

"El tema de la falta de oportunidades se da desde el gobierno, por el gobierno no ha tenido políticas públicas claras con nosotros, para implementarla a la población afrodescendiente $y$ de víctimas, supuestamente se llenan la boca diciendo que se van a implementar las politicas con enfoque diferencial para nuestras comunidades afrodescendientes, organizadas, pero mentira cuando llegamos al margen de todo simplemente nos hacen perder el tiempo" E7LD_MLaComadre.

Al respecto autores como (Carballeda, 2008) argumentan que un abordaje transversal desde las políticas públicas requiere de una aproximación al conjunto de problemas, necesidades, padecimientos y expectativas experimentados por una persona. Surge entonces en este punto la importancia de acceder a la trayectoria, historia de vida, potencialidades y perspectivas del sujeto; de esta forma, se visibiliza el desconocimiento que existe de las condiciones de las mujeres víctimas de desplazamiento que se encuentran asentadas en el Distrito, aspectos que se ven reflejados en la ausencia de programas que contribuyan al fortalecimiento y la generación de ingresos específicos para las mujeres afrocolombianas.

\section{Sin garantías para la generación de ingresos de las mujeres afrocolombianas}

Por último, la falta de implementación de acciones estratégicas que se reflejan en la ausencia de instituciones, visibilizando la falta de articulación, aspectos que se proyectan desde la descontextualización de las acciones, en términos de que no han sido efectivas, ni coherentes las acciones, desde el punto de vista de apoyo financiero para los proyectos productivos; ahora bien, desde este aspecto se destaca que ellas como colectivo de mujeres afrodescendientes han adelantado que aporten una contextualización de las necesidades y condiciones de la comunidad, 
sin embargo, al momento de realizar acciones a nivel distrital y departamental estas no se encuentran proyectadas con los procesos de contextualización realizados desde los intereses del colectivo.

"Aquí no hay garantías para estos programas de generación de ingresos, porque no los tienen dentro del Plan de Desarrollo, ni los tienen dentro del Plan de Acción Territorial (...) mientras no los tengan en esos planes no se puede decir que aquí va a funcionar una generación de ingresos" E9_MLaComadre

"Como experiencia es un poco complicado porque los programas que ha implementado el Distrito, son totalmente distintos a los programas desde la perspectiva que la unidad los ha propuesto” GF3_MLaComadre

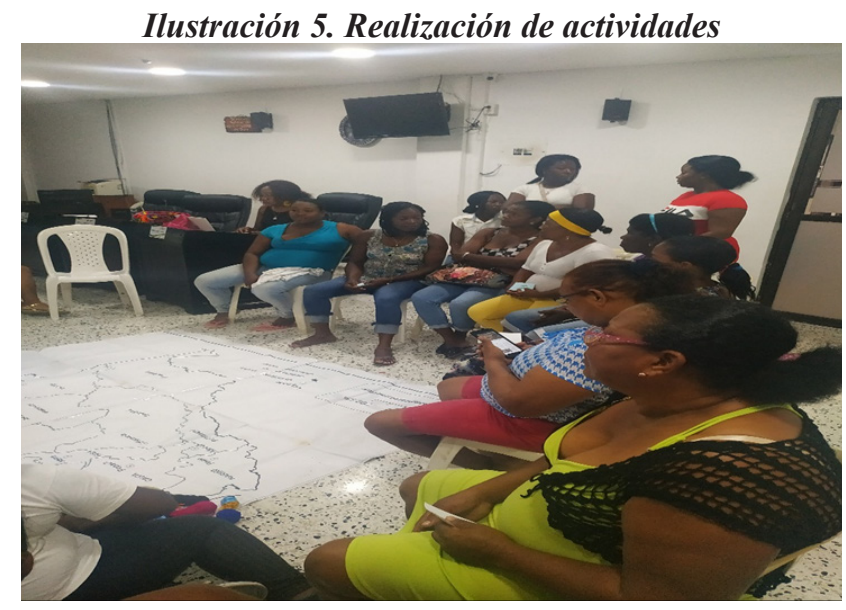

Fuente: autoras de la investigación

De igual forma, y con relación a los postulados anteriores realizados de acuerdo a la Política Pública para la inclusión efectiva de la población afrocolombiana en el Distrito de Cartagena, es importante destacar que no es posible establecer un nivel de avance de la política pública, teniendo en cuenta que no define indicadores que permitan su medición o evaluación y determinar el impacto hacia la población afrodescendiente víctima de desplazamiento forzado asentada en la ciudad de Cartagena.

\section{Limitaciones y retos de la generación de ingresos desde la percepción del Colectivo La Comadres, los funcionarios públicos y académicos}

Por lo tanto, cabe resaltar las limitaciones identificadas por parte de las mujeres, además de otros actores participantes como los funcionarios públicos y académicos sobre la implementación de la política pública de víctimas del desplazamiento, estas se encuentran directamente relacionadas con irregularidades en el Distrito, el presupuesto y la asignación de recursos, debilidades y fallas evidenciadas desde su implementación.

1. Las irregularidades en el Distrito se relacionan con los procesos de gobernanza débiles que ha vivenciado la ciudad de Cartagena en los últimos años, afectados por el cambio constante de gobernantes y las suspensiones de los mandatos administrativos, sumado a los procesos de corrupción continua que ha vivido la ciudad, procesos que en términos de gobernabilidad se asocian a la poca eficacia y eficiencia de la gestión pública Distrital.

"La corrupción que se ha llevado aquí en Cartagena ha hecho parte de todo este proceso de politica de estabilización en todo lo que ha pasado, en cuanto a los procesos organizativos que se han manejado en el distrito. Así que no hay que desconocer que parte de eso ha sido el fracaso de muchas cosas que no hemos podido lograr que se ejecuten como debieran de hacerse”E6LD_MLaComadre.

La ciudad carece de un "Arreglo Institucional ", es decir de un conjunto de normas y/o reglas transparentes, estables que garanticen la interacción entre los diferentes actores ciudadanos y reduzcan la incertidumbre en el relacionamiento público-privado. En este apartado se precisa como la gestión pública distrital está soportada en una cultura arraigada de la informalidad y la improvisación, asociado a una discontinuidad en los planes de desarrollo y programas ejecutados, 
evidenciando que la ciudad no ha podido construir un "Sistema de Planeación Distrital", concebido como la instancia que armoniza y regula la actuación de los actores públicos y privados sobre el territorio distrital, en la perspectiva de construir una Cartagena ordenada, equitativa, sostenible y competitiva, que garantice la producción de bienes y servicios a la población localizada en su jurisdicción. (Amaranto, Román, \& Jiménez, 2010, pág. 44)

2. Desde los discursos de los actores participantes a las limitaciones relacionadas con los procesos de corrupción se le suman la falta de presupuesto y destinación de recursos para emprender acciones $y$ desarrollar los proyectos focalizados a las mujeres afrocolombianas, de esta manera, las mujeres del colectivo, funcionarios y académicos entrevistados coinciden en su visión sobre los limitantes y obstáculos que desde la administración han tenido las mujeres afrodescendientes para la materialización de acciones y estrategias concretas encaminadas a la destinación de recursos para la población.

"El tema de los recursos impone una gran brecha que consiste en conciliar los plasmado en la politica con lo que efectivamente se realiza, dejando por fuera necesidades que han sido priorizadas en la formulación" E11A_MAs.UDC

"La posición de todos los gobernantes que han pasado de turno, que han dicho que supuestamente ellos tienen la voluntad, pero no hay recursos, pero mientras no haya recursos ellos no pueden hacer absolutamente nada. Se lavan las manos como Pilatos" E4LD_MLaComadre

3. Finalmente, las limitaciones que se hacen más complejas son las resaltadas por los funcionarios públicos, y que se evidencian desde la poca o nula participación de las mujeres afrodescendientes en la construcción de la política pública y en la toma de decisiones para las estrategias direccionadas para la generación de ingresos, orientadas a potenciar las habilidades y fortalezas que las mujeres afro descendiente tiene adquiridas en relación a su saberes ancestrales y vocación productiva como elementos de garantías de derechos en sus territorios.

"Se debe tener en cuenta que para la implementación de estas políticas publica en su fase de formulación se debió contar con las voces de las mujeres afro-descendientes no solo por mandato institucional y legal que ya está, sino de las mujeres afrodescendientes asentadas en el territorio quienes tienen la visión clara y compartida de las necesidades" E12F_MCoord

En este apartado, es importante precisar que desde la ley se establece la participación de las víctimas como el ejercicio que estas hacen del derecho a la participación a través del uso y disposición real y material de los mecanismos democráticos y los instrumentos previstos en la constitución y las leyes; de igual forma, de acuerdo al decreto 1084 de 2015, se entiende por participación aquel derecho de las víctimas a informarse, intervenir, presentar observaciones, recibir retroalimentación y coadyuvar de manera voluntaria, en el diseño de los instrumentos de implementación, seguimiento y evaluación de las disposiciones previstas en la Ley 1448 de 2011 y los decretos Ley para grupos étnicos víctimas, y los planes, programas y proyectos implementados para fines de materializar su cumplimiento. (Ministerio del Interior, 2016)

Ahora bien, las apuestas para el fortalecimiento de las políticas públicas en términos de generación de ingresos, desde las narrativas y experiencias de los actores que participaron en el proyecto de investigación se conciben con relación a tres aspectos fundamentales: el primero se relaciona con el respaldo y apoyo a las iniciativas presentadas por el colectivo La Comadre frente a las entidades competentes, a fin de que, las estrategias implementadas desde el Distrito sean contextualizadas a sus necesidades, 
y que no se plantean a priori a los procesos que desde la comunidad se inician. De igual forma, se resaltan las estrategias focalizadas desde enfoques integrales que aborden los componentes territoriales y diferenciales en relación a las particularidades de la población afrodescendiente, que permitan potenciar las capacidades de los territoritos $\mathrm{y}$ en el restablecimiento y goce efectivo de los derechos humanos.

"La implementación de una estrategia integral de formación a partir de sus intereses y habilidades personales, acompañado de la sostenibilidad financiera y asesorías para el éxito de sus emprendimientos o ideas de negocio” E16F_HRpL

"El avance en términos de generación de ingresos para que la cosa pueda andar es que realmente todas esas propuestas que nosotros como víctimas, como afrodescendientes les hemos presentado al Gobierno, ante la Alcaldía Local y Distritales se tuvieran en cuenta y se pudieran empezar a gestionar" E3LD_MLaComadre

Finalmente, el último aspecto se relaciona con el goce efectivo de los derechos de las mujeres víctimas, de manera que para el colectivo La Comadre es fundamental e importante el reconocimiento de las mujeres afrodescendientes como víctimas del conflicto armado y del desplazamiento forzado para garantizar el restablecimiento de sus derechos desde un accionar integral; al respecto (Arango, 2016) plantea que el concepto de goce efectivo de los derechos es de central importancia en la realización de los principios, derechos y deberes constitucionales. $\mathrm{Su}$ clarificación para el caso de la población víctima de desplazamiento forzado en Colombia es asunto de suma urgencia, supuesto que se trata de personas, en su mayoría mujeres y menores de edad que han sido golpeadas duramente por el conflicto armado y, en tal condición, gozan de la especial protección del Estado colombiano.

"Se debe ejercer una pedagogía tanto en los funcionarios como en las mujeres afrodescendientes víctimas del conflicto, atendiendo a la explicación de la necesidad de reconocer que estas medidas son de carácter integrar y que no solamente las vamos a minimizar a recibir la plata o los insumos para un negocio o recibir solamente el dinero para montar un negocio etc. Se debe dar un acompañamiento psicosocial, acompañamientojurídico, un acompañamiento académico a estas mujeres para presentarles la importancia del reconocimiento de estos derechos y para presentarle la importancia que estos items hacen parte de la reparación integral a las víctimas” GF3_MLaComadre

También, es importante resaltar que en el marco y construcción de nuevos escenarios de paz para el país, el colectivo de mujeres afrodescendientes representa un papel activo desde acciones políticas que han gestado, encaminadas a promover iniciativas de restablecimiento de derechos para la población víctima del desplazamiento forzado asentada en la ciudad de Cartagena, pero si bien, se han direccionado políticas públicas, planes, programas y proyectos que pueden ser beneficiosos para mejorar la calidad de vida de las mujeres afrocolombianas, estas iniciativas no transcienden a escenarios reales y no se alcanza su materialización en acciones concretas.

De esta forma, y con relación a los aspectos socioeconómicos se concluye que, las acciones desarrolladas a fin de garantizar una generación de ingresos para las mujeres afrocolombianas asentadas en el Distrito de Cartagena continuaran siendo descontextualizadas, al no tener suficiente conocimiento de las condiciones que representan a dicha población en términos de actividad laboral, formación, educación y actitudes vocacionales; por lo que, la implementación de acciones descontextualizadas, es muestra de las limitaciones y el proceder desarticulado por parte del Distrito para que las mujeres en su triple condición de víctimas, desplazadas y afrodescendientes continúen representando un accionar revictimizante sin garantías de 
restablecimiento de derechos.

\section{CONCLUSIONES}

Los hallazgos de la investigación dan cuenta de la importancia de precisar acciones específicas orientadas hacia la generación de ingresos de las mujeres afrocolombianas desplazadas, teniendo en cuenta que hasta el momento las acciones instauradas en el Distrito de Cartagena se visibilizan solo como soluciones emergentes hacia una problemática que es considerada de carácter estructural, de esta forma, es necesario proyectar y visionar políticas públicas que desde su diseño y ejecución atiendan realmente las necesidades económicas y sociales de las mujeres afrocolombianas asentadas en la ciudad.

De igual forma, es fundamental que desde la administración distrital se promuevan acciones tendientes a conocer de manera integral la situación y condición de las mujeres afrocolombianas que se encuentran asentadas en la ciudad de Cartagena de Indias, teniendo en cuenta que, es una realidad social que se complejiza en su abordaje, si desde el Distrito aún se continúan tomando como referente las cifras del año 2005, cuando se realizó el último censo poblacional, motivo por el cual para el Distrito es vital trabajar en la construcción de una línea base actualizada, a fin de proyectar y orientar estrategias de empleo y generación de ingresos con las mujeres afrocolombianas víctimas de desplazamiento forzado de forma contextualizada y acorde con su realidad social, económica y cultural.

De manera que, es fundamental tener presente y reconocer que las organizaciones de mujeres han surgido de procesos emancipatorios $\mathrm{y}$ liberatorios que les han permitido a las mujeres afrocolombianas fortalecer y dar continuidad a las acciones en defensa de sus derechos, a partir de gestiones que buscan incidir significativamente en los procesos de participación y construcción política para la reivindicación de derechos y acciones afirmativas para las víctimas del desplazamiento forzado, incidiendo desde su participación colectiva y política en procesos a nivel distrital y nacional.

Sin embargo, desde este aspecto es importante problematizar que aun cuando se han logrado múltiples avances y reconocimiento de los derechos de la población afrocolombiana y que desde los procesos organizativos de mujeres afrocolombianas se ha incidido de manera significativa fortaleciendo y promoviendo avances en términos de incidencia política, todavía queda mucho camino por recorrer para que lo decretado no se traduzca en letra muerta, sino que realmente se proyecten acciones y estrategias para la reivindicación de derechos en pro de mejorar su calidad de vida y la de sus familias.

Por lo anterior cabe entonces destacar que entre los aspectos a futuro y como una situación poco deseable se visibiliza que las mujeres afrocolombianas deberán continuar emprendiendo sus caminos y representaciones desde una lucha que deben gestar solas, teniendo en cuenta que, el esfuerzo por visibilizar sus posiciones se genera frente a un gobierno que se ha caracterizado por cerrar los ojos frente a las exigencias y las necesidades de las mujeres afrocolombianas víctimas de desplazamiento forzado.

Sin embargo, para que esta situación descrita con anterioridad no se continúe evidenciando en la realidad social de las mujeres afrocolombianas asentadas en el Distrito de Cartagena es pertinente que desde la institucionalidad se fortalezcan los procesos con la intención de responder de manera eficazy eficiente a la realidad socioeconómica de la población afrocolombiana desplazada en especial de las mujeres, formulando políticas públicas y direccionando programas que respondan a un enfoque étnico de género y diferencial, contextualizado a la realidad y a las necesidades específicas de las mujeres afrocolombianas.

De esta manera, se busca con el proceso de investigación realizado contribuir de manera significativa a las apuestas y movilizaciones 
del colectivo para considerar a las mujeres afrocolombianas como sujetas de reparación colectiva, además de resaltar el posicionamiento e incidencia en los diferentes escenarios políticos.

\section{DISCUSIÓN}

Desde la academia se hace fundamental apoyar la visibilización y el trabajo de los colectivos de mujeres afrocolombianas en relación a la labor significativa que vienen implementando en diversos escenarios del país en pro del mejoramiento de sus condiciones y calidad de vida, y de igual forma, la garantía y respaldo de sus derechos desde el ámbito legal para el reconocimiento como grupo poblacional como sujetas de reparación colectiva e integral a raíz de los hechos ocurridos en el marco del desplazamiento forzado y el conflicto armado colombiano.

De esta manera, visibilizar sus experiencias, las acciones que vienen emprendiendo y los obstáculos a los que se enfrentan permite desde lo social comprender la realidad social a la que se enfrentan y desde el ámbito jurídico implementar de manera más eficiente la normatividad y contribuir a frenar los vacíos legales para que como colectivo se les pueda garantizar la reivindicación de sus derechos económicos, políticos, sociales y culturales.

Así mismo, apostar por estos procesos de investigación permiten desde la academia generar espacios de reflexión y producción de conocimiento en relación a las categorías de análisis dispuestas, realizando contribuciones que favorecen el trabajo en relación a las políticas públicas, la generación de ingresos y la visibilización de la realidad de las mujeres afrocolombianas; de igual forma, los procesos de investigación direccionados en esta línea contribuyen y aportan significativamente a los procesos de intervención desde las instituciones estatales en especial en el Distrito de Cartagena, debido a que visibiliza la realidad y los sentires de las mujeres víctimas entorno a las estrategias para la generación de ingresos implementadas.

\section{NOTA}

El presente artículo es producto derivado de la tesis de maestría Percepciones del Colectivo "La Comadre" en torno a la Estrategia de Generación de Ingresos de la Política Pública de Víctimas del Desplazamiento en la ciudad de Cartagena, periodo 2016 al 2019, realizada en el año 2020 para optar al título de Magíster en Conflicto Social y Construcción de Paz de la Universidad de Cartagena.

\section{REFERENCIAS}

1. Alcaldía de Cartagena . (2007). Plan Integral Único para la prevención y atención a la problemática del desplazamiento forzado en el Distrito de Cartagena (PIU).

2. Alcaldía de Cartagena . (2016). Plan de Acción Territorial (PAT) 2016 - 2019 Víctimas Integradas.

3. Amaranto, D., Román, R., \& Jiménez, J. (2010). La población desplazada en Cartagena de Indias: alcances y limitaciones de la política pública. Revista Palobra, " palabra que obra", 1(11), 40-56.

4. Arango, R. (2016). El concepto de goce efectivo de derechos. En Realizando los derechos: Su filosofía y práctica en América Latina. Instituto de Estudios Constitucionales del Esatado de Querétaro.

5. Becerra, L. M. (2019). La Coordinación de Mujeres Afrocolombianas Desplazadas en Resistencia "La COMADRE", de la Asociación Nacional de Afrocolombianos Desplazados AFRODES.

6. Carballeda, A. J. (2008). Problemáticas sociales complejas y políticas públicas. CS, 261-272.

7. Castillo, M., Segrera, A., \& León, R. (2018). Resignificando identidad en un territorio urbano que transita hacia una convivencia no violenta. Estudio de caso sector las Vegas del barrio Nelsónn Mandela, de la ciudad de Cartagena de Indias 2015-2017 . (Doctoral dissertation, Universidad de Cartagena). 
8. Centro Nacional de Memoria Histórica . (2015). Una Nación Desplazada. Informe Nacional del Desplazamiento Forzado en Colombia. Bogotá: CNMH - UARIV.

9. Congreso de la República . (20 de Junio de 2005). Decreto 2050. Por el cual se convoca al Congreso de la República a sesiones extraordinarias . Bogotá D.C.

10. Corte Constitucional . (26 de Enero de 2009). Auto 005. Protección de derechos fundamentales de la población afrodescendiente víctima del desplazamiento forzado en el marco de superación del estado de cosas inconstitucional declarado en sentencia T-025/04. Bogotá D.C. .

11. Corte Constitucional. (22 de Enero de 2004). Sentencia T-025. A GENCIA OFICIOSA EN TUTELA - Asociaciones de Desplazados / AGENCIA OFICIOSA EN TUTELA - Condiciones para que las asociaciones de desplazados interpongan la acción. Bogotá D.C.

12. Corte Constitucional. (14 de Abril de 2008). Auto 092. Adopción de Medidas para la Protección de Mujeres Víctimas del Desplazamiento Forzado por Causa del Conflicto Armado. Bogotá D.C. .

13. Corte Constitucional. (21 de Mayo de 2013). Auto 098. Sentencia de la Corte Constitucional en Materia de Desplazamiento Forzado. Bogotá D. C. .

14. Corte Constitucional. (27 de Enero de 2015). Auto 009. SALA ESPECIAL DE SEGUIMIENTO SENTENCIA T-025 DE 2004. Bogotá D.C.

15. DNP. (2020). Informe Presupuestal de la Política Pública Dirigida a la Población Víctima del Desplazamiento Forzado 2019-2020. 2-81: Minihacienda .

16. Jiménez, W. G., \& Ramírez, C. A. (2008). Gobierno y Política Públicas. Escuela Superior de Administración Pública .

17. Mata, L. D. (28 de mayo de 2019). Investigalia. Obtenido de El enfoque cualitativo de investigación: https://investigaliacr.com/investigacion/el-enfoque-cualitativo-de-investigacion/\#: :text=La\%20 investigaci\%C3\%B3n\%20cualitativa\%20asume \%20una,parte\%20de \%20las\%20realidades $\% 20$ estudiadas.

18. Ministerio del Interior. (2016). Orientaciones para la incorporación de la estratégica de la política pública de víctimas y restitución de tierras en los planes territoriales de desarrollo 2016-2019.

19. Natalia, B., \& Inciarte, A. (2012). Fenomenología y hermenéutica: dos perspectivas para estudiar las ciencias sociales y humanas. Multiciencias , 199-205.

20. PNUD . (2010). Hechos del Callejón Número 51. Las Mujeres como Constructoras de Paz. Programa de las Naciones Unidas para el Desarrollo.

21. Puello, A. D., Romero, R. R., \& García, J. J. (2010). La población desplazada en Cartagena de Indias: alcances y limitaciones de la política pública. . Revista Palobra, 40-56.

22. Rojas, J. C. (2016). Etapas del conflicto armado en Colombia: hacia el posconflicto. Latinoamérica. . Revista de Estudios Latinoamericanos., 227-257.

23. RUV. (31 de Mayo de 2020). Unidad de Victimas. Obtenido de Registro Unico de Victimas: https:// www.unidadvictimas.gov.co/es/reportes

24. SNAIPD. (2011). PRONUNCIAMIENTO DEL GOBIERNO NACIONAL SOBRE LA POLÍTICA PÚBLICA PARA LA POBLACIÓN VÍCTIMA DEL DESPLAZAMIENTO FORZADO POR LA VIOLENCIA. Bogota, Colombia.: Sistema Nacional de Atencion Integral a la Poblacion Desplazada. 